\title{
NECROTISING ENTEROCOLITIS AND SYSTEMIC INFLAMMATION: PLACENTAL PATHOLOGY AND HAEMATOLOGICAL MARKERS OF MORTALITY
}

\author{
S. Baastad ${ }^{1}$, R. McCarthy ${ }^{2}$, R. Lee ${ }^{3}$, F. O'Hare ${ }^{2}$, E. Mooney ${ }^{4}$, T. Grant ${ }^{5}$, E. Molloy ${ }^{2}$ \\ ${ }^{1}$ Royal College of Surgeons, ${ }^{2}$ Department of Neonatology, National Maternity Hospital, ${ }^{3}$ UCD School of \\ Medicine and Medical Sciences, University College Dublin, ${ }^{4}$ Pathology, National Maternity Hospital, \\ ${ }^{5}$ School of Public Health and Population Science, University College Dublin, Dublin, Ireland
}

Background and aims: Necrotizing enterocolitis (NEC) is a devastating disease that affects premature neonates. Associated mortality has not changed appreciably over the past several decades. The underlying aetiology of NEC remains elusive, although bacterial colonization of the gut, formula feeding, and perinatal stress have been implicated as risk factors.

We aimed to characterize clinical indicators predicting mortality from NEC.

Methods: A prospective database of infants with Necrotising enterocolitis Bell's staging $\geq 2$ was used to identify eligible infants. Full blood count (FBC) and C-reactive protein (CRP) before, at and after the diagnosis of NEC as well as placental pathology and Bayleys scores were evaluated.

Results: 68 infants were included with an age at onset of NEC of 18+/-14 days and 25 infants died. Chorioamnionitis was significantly increased in non-survivors. IT ratio, Platelets (Plts) and CRP were also significantly altered between survivors and no-survivors. Prophylactic indomethacin use and intestinal perforation were increased in non-surviving infants. Antenatal steroid use, mode of delivery, Apgars, age starting feeds and presence of a PDA did not differentiate survivors and non-survivors. Surviving infants with perforated NEC had a significantly decreased motor and cognitive scores at 2 years.

Conclusions: Prediction of infants with NEC who will develop intestinal perforation and have an increased mortality risk would be useful in clinical practice to allow early surgical referral. Persistent alterations in inflammatory markers may signify infants who may have increased risk of mortality. 